\section{Mechanisms of axon-glial injury of the optic nerve}

\section{A Compston}

think merely in terms of demyelination as the sole consequence of brain inflammation. Rather, symptom onset and recovery must be understood in terms of altered structure and function in the entire myelinated oligodendrocyte-axon unit.

Mitochondrial disease and the optic nerve

Genetic factors are implicated in the aetiology of multiple sclerosis. The best characterized region of interest is the major histocompatibility complex encoded at chromosome 6p21. Possession of the DR15 susceptibility allele in individuals with optic neuritis increases the risk of conversion to multiple sclerosis. ${ }^{1}$ This merely reflects the relationship of optic neuritis to multiple sclerosis. Potentially more revealing is the identification of a multiple sclerosis-like illness in women, presenting with bilateral sequential optic neuropathy and later developing symptoms consistent with demyelination outside the visual system, in whom there are mutations of mitochondrial DNA (Harding's disease). ${ }^{2}$ This immediately suggests a genetic basis for selective involvement of the optic nerve in demyelinating disease. Around $45 \%$ of individuals with pathological mutations of mitochondrial DNA have clinical manifestations outside the visual system. ${ }^{3}$ But mitochondrial mutations are rarely identified with systematic screening of unselected patients, although the yield is higher in patients with severe visual failure complicating demyelinating disorder of the central nervous system. What remains unresolved is whether the mutation of mitochondrial DNA in Harding's disease directs the process of brain inflammation onto a particular site-constituting selective tissue vulnerability - or merely represents the chance occurrence of relatively mild multiple sclerosis and Leber's hereditary optic neuropathy.
Received: 4 September 2003

Accepted: 4 September 2003

\begin{abstract}
As the symptoms are immediately apparent to affected individuals, optic neuritis provides special opportunities for clinical and experimental analysis of brain inflammation. The spectrum of inflammatory optic neuropathy includes conditions confined to the optic nerve and those also affecting other parts of the central nervous system. It follows that the optic nerve provides a general window on the aetiology, mechanisms of symptom onset and recovery, and pathogenesis of inflammatory brain disease. No longer is it appropriate to

Keywords: optic nerve; multiple sclerosis; inflammation; demyelination; axonal injury; growth factors; remyelination
\end{abstract}

Correspondence:

CB2 2QQ, UK

Fax: + 441223336941

E-mail: DASCompston@ aol.com 
In neuromyelitis optica (Devic's disease), demyelination affects the spinal cord and optic nerve. These sites may be affected serially and in either order. Magnetic resonance imaging (MRI) does not typically show lesions at other sites in the central nervous system. ${ }^{4}$ Pathologically, the affected areas show inflammation with extensive necrosis and deposition of antibody and complement. ${ }^{5}$ Patients may respond to plasma exchange. ${ }^{6}$ However, the severe optic neuropathy occurring in the context of neuromyelitis optica among Caucasians is also not associated with mutations of mitochondrial DNA. ${ }^{7}$ Thus, mutations of mitochondrial DNA evidently do not easily account for the high frequency of optic nerve involvement. However, visual involvement in multiple sclerosis may generally be associated with the mitochondrial $\mathrm{K}$ and J haplogroups, suggesting that these serve as population markers of autosomal genes conferring selective tissue susceptibility to demyelinating disease. ${ }^{8}$

\section{Clinically isolated optic neuritis}

Optic neuritis is typically unilateral and transient but may later recur in the same or the other eye. A high proportion of affected individuals subsequently experience recurrent episodes of demyelination affecting different parts of the central nervous system. The risk is further increased for those with cranial MRI abnormalities at presentation. ${ }^{9}$ Serial clinical, electrophysiological, and imaging observations suggest a sequence in which inflammation leads to loss of vision in association with impaired saltatory conduction through the normally myelinated optic nerve. As inflammation subsides, conduction is restored and vision improves. ${ }^{10}$ But structural damage may persist despite functional recovery. There is a reduction in calibre of the optic nerve following a transient episode of optic neuritis, indicating a loss of axonal density. ${ }^{11}$ Yet vision improves rapidly and serial studies provide some evidence for systematic reduction in latency of the evoked potential consistent with remyelination of surviving axons. ${ }^{12}$

Thus, the study of optic neuritis has the potential to illuminate the basic principles of symptom onset and recovery, the dynamics of myelin and axonal injury, and the switch from clinically isolated to distributed demyelinating disease. The evidence suggests that (at least) three separate mechanisms of symptom onset and three related but somewhat different explanations for recovery interact to account for the complex pathogenesis of optic nerve and brain inflammation.

\section{Pathogenesis of brain inflammation}

The clinical course in multiple sclerosis is initially characterized by episodes with full recovery, later by attacks that leave persistent deficits and, as the frequency of new exacerbations eventually decreases, by the onset of secondary progression. Transition between these three stages is usually gradual and indistinct, making for many intermediate forms of the disease. This clinical course is the expression of focal tissue injury affecting the brain and spinal cord and resulting from the complex interplay of inflammation, axonal injury, demyelination, remyelination, astrocytosis, and tissue atrophy.

The central concept underlying ideas on the pathogenesis of multiple sclerosis is that the cascade of inflammatory events that culminates in demyelination of axons depends on the peripheral activation of $\mathrm{T}$ lymphocytes. Activated T cells express adhesion molecules on their surface and upregulate complementary molecules on the luminal surface of blood vessels, allowing them to cross the blood-brain barrier by diapedesis. Within the central nervous system, these T cells re-encounter specific antigen and set up an inflammatory process that resembles delayed-type hypersensitivity, dominated by lymphocytes and microglia. Various immunological effector mechanisms are initiated - cytotoxic T-cell proliferation, antibody production, and activation of microglia. These then act as antigen-presenting cells, increasing the expression of class II major histocompatibility antigens and amplifying the local inflammatory response. They engage opsonized myelin and oligodendrocytes, causing demyelination by cell-cell adherence and local release of inflammatory mediators. As a result, the myelin-oligodendroctye unit is damaged, saltatory conduction breaks down, and the symptoms of multiple sclerosis follow. But this is not the whole story. The impact of axonal injury has recently been revisited and more fully defined.

Clinical observations and related laboratory and experimental studies show that cytokines released by immune cells acting locally or in the systemic circulation induce transient conduction block - in the optic nerve and elsewhere. In the unusual setting of monoclonal antibody therapy targeting the CD52 antigen, lymphocytotoxicity leads to an acute cytokine release syndrome. This is associated with transient recurrence of many previously experienced symptoms and signs of multiple sclerosis. Since the onset is abrupt and severe but subsequent recovery is complete, it seems likely that cytokines cause acute electrical failure in pathways previously compromised by inflammation and demyelination. This interpretation is borne out by the demonstration of transient blindness and loss of conduction through the optic nerve in one patient who 
had recovered from a previous episode of optic neuritis. The subsequent deterioration in vision and loss of amplitude in the already delayed visual-evoked response were each restored within $24 \mathrm{~h}$. Other patients in this series experienced specifically different losses of function in association with cytokine release but with recovery over the same time course. ${ }^{13}$ The experimental evidence implicates donors of nitric oxide as the main factors responsible for these alarming but instructive clinical effects. Brief exposure of the exposed rat spinal cord produces reversible conduction block in normal or hypomyelinated axons. ${ }^{14}$ Conduction block in pathways affected by multiple sclerosis may also be caused by a pentapeptide (Gln-Tyr-Asn-Ala-Asp), which is able to block sodium channels and is present at increased concentration in cerebrospinal fluid from patients with multiple sclerosis. ${ }^{15}$ Hence, one mechanism of symptom onset is the direct effect of inflammatory mediators on conduction through myelinated, and more especially hypomyelinated, axons. It follows that, in situations where structural damage may not have occurred, recovery follows removal of inflammatory mediators reversing the functional deficit affecting intact myelinated axons.

Although axonal dysfunction is initially reversible, a separate and lethal sequence of calcium-dependent excitotoxic events follows more prolonged exposure to inflammatory mediators. Electrically active axons are especially vulnerable and show irreversible conduction block with axonal degeneration after prolonged exposure to nitric oxide. ${ }^{16}$ This analysis resonates with contemporary studies of axonal pathology in multiple sclerosis. Immunohistochemical staining for amyloid precursor protein confirms that axonal injury occurs as part of the acute demyelinating lesion. ${ }^{17}$ Axonal damage in acute inflammatory plaques is shown histologically ${ }^{18}$ and radiologically through reduction in the neuronal spectroscopic marker, $\mathrm{N}$-acetyl aspartate. ${ }^{19}$ Acutely transected axons undergo Wallerian degeneration over the subsequent 18 months, but this seems not to extend the lesion or shape the clinical deficit. ${ }^{20}$ This acute axonal damage mainly occurs early in multiple sclerosis and is correlated with the degree of inflammation. ${ }^{21}$

The circumstantial evidence suggesting that recently demyelinated axons are vulnerable to the inflammatory environment of acute lesions is supported by experimental studies. Soluble factors released by activated microglia impair mitochondrial (cytochrome oxidase) activity of neurons in vitro. ${ }^{22}$ Although this neuronal dysfunction is initially reversible, a separate and lethal sequence of events follows more prolonged exposure to microglial soluble factors. In cocultures of rat microglia and embryonic cortical neurones,
iNOS-derived nitric oxide alone is responsible for neuronal death from IFN $\gamma$ and activated microglia. Neurones allowed to mature in vitro remain sensitive to nitric oxide but are rescued by blocking NMDA-receptor activation, indicating that nitric oxide elicits an excitotoxic mechanism, most likely acting through neuronal glutamate release. Thus, similar concentrations of nitric oxide cause neuronal death by two distinct mechanisms: nitric oxide acts directly upon immature neurones but indirectly, via NMDA receptors, on more mature neurones. ${ }^{23}$

Intravenous methylprednisolone acutely suppresses nitric oxide release and might thereby protect neurones from the effects of inflammation, thus reducing disability acquired from an individual relapse. Clinical trials of corticosteroid treatment in optic neuritis ${ }^{24,25}$ do not support this view, nor does the demonstration of continued atrophy in optic nerves, by MRI, after steroid treatment. ${ }^{11}$ However, the mean duration of symptoms before treatment was 6 days in the Optic Neuritis Treatment Trial, by which time the threshold for recovery from nitric oxide exposure may have been crossed leading to irreversible axonal injury. Support for the neuroprotective role of corticosteroids is provided by a clinical trial showing that regular pulsed methylprednisolone, while not influencing mechanisms that initiate relapses in multiple sclerosis, nevertheless reduces the consequences of each episode for axons and, perhaps, impedes the transition to disease progression. ${ }^{26}$

However, despite these mechanisms of structural tissue injury, function may recover even after prolonged exposure to inflammatory mediators. One potential mechanism is neuronal plasticity. Oligodendrocytes normally determine the distribution and clustering of sodium and potassium channels at nodes of Ranvier. ${ }^{27}$ Specifically, oligodendrocyte-derived soluble factors induce clustering of the $\mathrm{Na}(\mathrm{v}) 1.2$, whereas myelin is required for clustering of the $\mathrm{Na}(\mathrm{v}) 1.6$ channel. ${ }^{28}$ With maturation, the diffuse distribution of $\mathrm{Na}(\mathrm{v}) 1.2$, channels is rationalized, being retained along the myelinated segments but replaced by $\mathrm{Na}(\mathrm{v}) 1.6$ channels at the nodes. ${ }^{29}$ These arrangements are lost in demyelinated axons, sodium channels again becoming distributed along the naked axon leading to a variety of alternative mechanisms for propagation of the nerve impulse. ${ }^{30}$ While imperfect and with a reduced safety factor for conduction, these rearrangements do at least restore function despite some persistent structural alterations in axon-glial arrangements. There is also evidence that the threat to sophisticated function from inflammatory injury of the optic nerve leads to changes in cortical receptor field activities - presumably resulting from loss of inhibition releasing areas of cortex to capture and modify impulses arriving upstream of the injured optic nerve. ${ }^{31}$ 
While details of the role and mechanism of opening up these cortical receptor fields as part of the response to optic neuritis remain speculative, the probability of widespread neuronal and cortical plasticity as part of the compensatory mechanism seems likely and consistent with the evidence from functional brain imaging.

A number of clinical trials have now made the point that the suppression of inflammation in chronic multiple sclerosis rarely does much to limit the accumulation of disability through sustained progression. ${ }^{32}$ One interpretation of these observations is that axonal loss and inflammation are entirely independent pathologies. ${ }^{33}$ The epidemiological jury is still out on whether there is a correlation between the early inflammatory load and subsequent clinical course. But the experimental evidence suggests a direct relationship. Most axonal loss is seen in secondary progressive multiple sclerosis. Embryonic (rat) cortical neurons, cocultured with purified oligodendrocytes at different developmental stages, and with oligodendrocyte-conditioned medium, show a marked increase in cell number providing experimental evidence that myelin or glia provide survival factors for neurones. ${ }^{34}$ Neutralizing antibodies to IGF-1, but not other candidate trophic factors, block this soluble survival effect of oligodendrocytes. Cells of the oligodendrocyte lineage produce IGF-1 and recombinant IGF-1 promotes neuronal survival under identical conditions. Oligodendrocyte-conditioned medium increases levels of phosphorylated Akt within neurons. Neurons exposed to the oligodendrocyteconditioned medium show increased neurite and axonal length, detected by antibodies to phosphorylated neurofilaments, and activation of the MAPkinase/Erk pathway. Erk inhibition reduces oligodendrocytemediated enhancement of axonal length, but, unlike inhibition of $\mathrm{PI}_{3}$ kinase, this has no effect on neuronal survival. ${ }^{35}$ This in vitro evidence suggests that cells of the oligodendrocyte lineage support neuronal survival by both contact-mediated and soluble mechanisms, and that IGF-1 contributes this effect through the PI3kinase/Akt signalling pathway. Conversely, differentiated oligodendrocytes increase neurofilament phosphorylation and axonal length due to an effect of glial cell-derived nerve growth factor (GDNF) acting through the MAPkinase/Erk pathways. Loss of these mechanisms may explain the chronic axonal attrition characteristic of multiple sclerosis.

Taken together, the phases of symptom onset, recovery, persistence, and progression in isolated and multiple lesions are: functional impairment with intact structure due to nitric oxide-related mechanisms; demyelination and axonal injury with recovery from plasticity and remyelination; and chronic axonal loss due to failure of enduring remyelination.
The therapeutic opportunities and challenges of this analysis are self-evident. The critical issue is the relationship between neuronal injury and brain inflammation. If axons degenerate solely because of the metabolic and physiological consequences of prolonged demyelination then therapies that reduce demyelination should prevent disability progression. Early suppression of inflammation is also logical if axons are transected in acute inflammatory plaques, and degenerate chronically through loss of trophic support from cells of the oligodendrocyte lineage. But if axonal loss and inflammation are fully independent processes, immunotherapy may not be capable of influencing progression of disability however early this is deployed.

Experimentally, endogenous remyelination restores conduction and function both in young and adult nervous systems. ${ }^{36,37}$ The lesions of multiple sclerosis contain oligodendrocyte progenitors, but these seem unable to engage naked axons usefully. ${ }^{38,39}$ Endogenous remyelination is limited to the acute phase of brain inflammation during which manipulation of mechanisms involved in receptor-ligand growth factor interactions might envigorate these indolent progenitors and improve remyelination. The choice and delivery of such interventions aimed at enhancing remyelination are problematic. Pragmatically, one option is to wait until a therapy is available that can be given systemically and simultaneously reaches all affected parts of the central nervous system. The alternative is first to prove that structure and function can be usefully restored in a single informative lesion before tackling the secondary task of making this intervention diffusely available in the central nervous system. Until recently, stereotactic delivery seemed the only possible option. However, the situation may have changed with the demonstration of remyelination and acute neuroprotection following intravenous delivery of neural stem cells. Murine neural stem cells injected intravenously into animals with experimental allergic encephalomyelitis express the adhesion molecules needed to adhere to and penetrate the blood-brain barrier. ${ }^{40}$ Evidently, they successfully seek out areas of demyelination and, once in place, differentiate and remyelinate surviving axons, not only achieving structural repair but also restoring physiological conduction and motor function in affected animals. Furthermore, remyelination protects axons from the degeneration that otherwise follows demyelination. These results suggest a remarkable set of attributes for cells that have not been manipulated in vitro. While providing hope and raising expectations, many practical, clinical, ethical, and biological problems remain to be overcome before remyelination can become a reality for individual patients with multiple sclerosis. 


\section{References}

1 Hauser SL, Oksenberg JR, Lincoln R, Garovoy J, Beck RW, Cole SR et al. Interaction between HLA-DR2 and abnormal brain MRI in optic neuritis and early MS. Optic Neuritis Study Group. Neurology 2000; 54: 1859-1861.

2 Harding AE, Sweeney MG, Miller DH, Mumford CJ, Kellar-Wood H, Menard D et al. Occurrence of a multiple sclerosis-like illness in women who have a Leber's hereditary optic neuropathy mitochondrial DNA mutation. Brain 1992; 115: 979-989.

3 Riordan-Eva P, Sanders MD, Govan GG, Sweency MG, Da Costa J, Harding AE. The clinical features of Leber's hereditary optic neuropathy defined by the presence of a pathogenic mitochondrial DNA mutation. Brain 1995; 118: 319-337.

4 Wingerchuk DM, Hogancamp WF, O'Brien PC, Weinshenker BG. The clinical course of neuromyelitis optica (Devic's synsrome). Neurology 1999; 53: 1107-1114.

5 Lucchinetti CF, Mandler R, McGavern D, Bruck W, Gleich $\mathrm{G}$, Ransohoff RM et al. A role for humoral mechanisms in the pathogenesis of Devic's neuromyelitis optica. Brain 2000; 125: $1450-1461$.

6 Weinshenker BG, O'Brien PC, Petterson TM, Noseworthy $\mathrm{JH}$, Lucchinetti $\mathrm{CF}$, Dodick DW et al. A randomized trial of plasma exchange in acute central nervous system inflammatory demyelinating disease. Ann Neurol 1999; 46: 878-886.

7 Kalman B, Mandler RN. Studies of mitochondrial DNA in Devic's disease revealed no pathogenic mutations, but polymorphisms also found in association with multiple sclerosis. Ann Neurol 2002; 51: 661-662.

8 Reynier P, Penisson-Besnier I, Moreau C, Savagner F, Vielle B, Emile J et al. mtDNA haplotyping J: a contributing factor of optic neuritis. Eur J Hum Genet 1999; 7: 404-406.

9 Brex PA, Gomez-Anson B, Parker GJ, Molyneux PD, Miszkiel KA, Barker GJ et al. Proton MR spectroscopy in clinically isolated syndromes suggestive of multiple sclerosis. J Neurol Sci 1999; 66: 16-22.

10 Youl BD, Turano G, Miller DH, Towell AD, MacManus DG, Moore SG et al. The pathophysiology of acute optic neuritis. An association of gadolinium leakage with clinical and electrophysiological deficits. Brain 1991; 114: 2437-2450.

11 Hickman SJ, Brex PA, Brierley CMH, Silver NC, Barker GJ, Scolding NJ et al. Detection of optic nerve atrophy following a single episode of unilateral optic neuritis by MRI using a fat saturated short echo fast FLAIR sequence. Neuroradiology 2001; 43: 123-128.

12 Brusa A, Jones SJ, Plant GT. Long-term remyelination after optic neuritis: a 2 year visual evoked potential and psychophysical serial study. Brain 2001; 124: 468-479.

13 Moreau T, Coles A, Wing M, Isaacs J, Hale G, Waldmann II, Compston A. Transient increase in symptoms associated with cytokine release in patients with multiple sclerosis. Brain 1996; 119: 225-237.

14 Redford EJ, Kapoor R, Smith KJ. Nitric oxide donors reversibly block axonal conduction: demyelinated axons are especially susceptible. Brain 1997; 120: 2149-2157.

15 Brinkmeier H, Aulkemeyer P, Wollinsky KH, Rudel R. An endogenous pentapeptide acting as a sodium channel blocker in inflammatory autoimmune disorders of the central nervous system. Nature Medicine 2000; 6: 808-811.
16 Smith KJ, Kapoor R, Hall SM, Davies M. Electrically active axons degenerate when exposed to nitric oxide. Ann Neurol 2001; 49: 470-476.

17 Ferguson B, Matyszak MK, Esiri MM, Perry VH. Axonal damage in acute multiple sclerosis lesions. Brain 1997; 120: 393-399.

18 Trapp BD, Peterson J, Ransohoff RM, Rudick R, Mork S, Bo L. Axonal transection in the lesions of multiple sclerosis. N Eng J Med 1998; 338: 278-285.

19 Davie CA, Barker GJ, Webb S, Tofts PS, Thompson AJ, Harding AE et al. Persistent functional deficit in multiple sclerosis and autosomal dominant cerebellar ataxia is associated with axon loss. Brain 1995; 118: 1583-1592.

20 Simon JH, Jacobs L, Kinkel RP. Transcallosal bands: a sign of neuronal tract degeneration in early MS? Neurology 2001; 57: 1888-1890.

21 Kuhlmann T, Lingfeld G, Bitsch A, Schuchardt J, Bruck W. Acute axonal damage in multiple sclerosis is most extensive in early disease stages and decreases over time. Brain 2002; 125: 2202-2212.

22 Nicholas RS, Compston A, Brown DR. Inhibition of tumour necrosis factor-alpha (TNFalpha)-induced NF-kappaB p52 converts the metabolic effects of microglial-derived TNFalpha on mouse cerebellar neurones to neurotoxicity. J Neurochem 2001; 76: 1431-1438.

23 Golde S, Chandran S, Brown GC, Compston A. Different pathways for iNOS-mediated toxicity in vitro dependent on neuronal maturation and NMDA receptor expression. J Neurochem 2002; 82: 269-282.

24 Optic Neuritis Study Group. The 5-year risk of MS after optic neuritis. Experience of the optic neuritis treatment trial. Neurology 1997; 49: 1404-1413.

25 Beck RW, Cleary PA, Anderson Jr MM, Keltner JL, Shults WT, Kaufman DI et al. A randomized, controlled trial of corticosteroids in the treatment of acute optic neuritis* The Optic Neuritis Study Group. N Eng J Med 1992; 326: 581-588.

26 Zivadinov R, Rudick RA, De Masi R, Nasuelli D, Ukmar M, Pozzi-Mucelli RS et al. Effects of IV methylprednisolone on brain atrophy in relapsing- remitting MS. Neurology 2001; 57: 1239-1247.

27 Mathis C, Denisenko-Nehrbass N, Girault JA, Borrelli E. Essential role of oligodendrocytes in the formation and maintenance of central nervous system nodal regions. Development 2001; 128: 4881-4890.

28 Kaplan MR, Cho MH, Ullian EM, Isom LL, Levinson SR, Barres BA et al. Differential control of clustering of the sodium channels $\mathrm{Na}(\mathrm{v}) 1.2$ and $\mathrm{Na}(\mathrm{v}) 1.6$ at developing CNS nodes of Ranvier. Neuron 2001; 30: 105-119.

29 Boiko T, Rasband MN, Levinson SR, Caldwell JII, Mandel G, Trimmer JS et al. Compact myelin dictates the differential targeting of two sodium channel isoforms in the same axon. Neuron 2001; 30: 91-104.

30 England JD, Gamboni F, Levinson SR, Finger TE. Changed distribution of sodium channels along demyelinated axons. Proc Nat Acad Sci USA 1990; 87: 6777-6780.

31 Werring DJ, Bullmore ET, Toosy AT, Miller DII, Barker GJ, MacManus DG et al. Recovery from optic neuritis is associated with a change in the distribution of cerebral response to visual stimulation: a functional magnetic resonance imaging study. J Neurol Neurosurg Psychiatry 2000; 68: 441-449.

32 Coles AJ, Wing M, Molyneux P, Paolillo A, Davie CM, Hale $\mathrm{G}$ et al. Monoclonal antibody treatment exposes three 
mechanisms underlying the clinical course of multiple sclerosis. Ann Neurol 1999; 46: 296-304.

33 Confavreux C, Vukusic S, Moreau T, Adeleine P. Relapses and progression of disability in multiple sclerosis. $N$ Eng $J$ Med 2000; 343: 1430-1438.

34 Wilkins A, Chandran S, Compston A. A role for oligodendrocyte-derived IGF-1 in trophic support of cortical neurons. Glia 2001; 36: 48-57.

35 Wilkins A, Majed H, Layfield R, Compston A, Chandran S. Oligodendrocytes promote neuronal survival and axonal length by distinct intracellular mechanisms: a novel role for oligodendrocyte-derived glial cell line-derived neurotrophic factor. J Neurosci 2003; 23: 4967-4974.

36 Barnett SC, Alexander CL, Iwashita Y, Gilson JM, Crowther J, Clark L et al. Identification of a human olfactory ensheathing cell that can effect transplant-mediated remyelination of demyelinated CNS axons. Brain 2000; 123: 1581-1588.

37 Kohama I, Lankford KL, Preiningerova J, White FA, Vollmer TL, Koesis JD et al. Transplantation of cryopreserved adult human Schwann cells enhances axonal conduction in demyelinated spinal cord. J Neurosci 2001; 21: 944-950.

38 Scolding N, Franklin R, Stevens S, Heldin CH, Compston A, Newcombe J. Oligodendrocyte progenitors are present in the normal adult human CNS and in the lesions of multiple sclerosis. Brain 1998; 121: 2221-2228.

39 Wolswijk G. Oligodendrocyte precursor cells in the demyelinated multiple sclerosis spinal cord. Brain 2002; 125: 338-349.

40 Pluchino S, Quattrini A, Brambilla E, Gritti A, Salani G, Dina $G$ et al. Injection of adult neurospheres induces recovery in a chronic model of multiple sclerosis. Nature 2003; 422: 688-694. 